\title{
Percepción sobre la violencia de género según el nivel educativo y el género
}

Fecha de recepción: 4/07/2014

Fecha de aceptación: 22/10/2014
${ }^{1}$ Francisco González Sala y ${ }^{2}$ Belén Mora Valero
'Universidad de Valencia

2Programa de Prevención de la Asociación de Voluntarios de Acogimiento Familiar

\section{resumen/ahstract:}

El presente estudio tiene como objetivo comparar diferentes variables relacionadas con la violencia de género entre estudiantes universitarios y de otros niveles educativos y el género. La muestra está formada por 247 estudiantes. Los resultados apuntan diferencias estadísticamente significativas en función del nivel educativo en las variables percepción de los tipos de maltrato, aprobación de la violencia y tolerancia de conductas de riesgo, entre otras. La percepción de los estudiantes no universitarios frente a los universitarios los coloca en una situación de mayor riesgo ante la misma. Con respecto al género las diferencias se dan en algunas formas de actuar y en la percepción de los tipos de maltrato y a su aprobación. Estos resultados han de servir para orientar en la implementación de programas de prevención de la violencia de género y de relaciones de pareja saludables dentro del contexto educativo, en especial en los niveles no universitarios.

This study aims to compare different variables related to gender violence among college students and other educational level sand gender. The sample consisted of 247 students. The results indicate significant differences in terms of educational level indicators on both how to cope with violence and considerations regarding gender violence. The university are not manifesting a degree of know ledge of gender violence less, putting them at a greater risk to the same. Regarding gender differences exist in some forms of acting and considerations regarding violence. These results will be used to guide the implementation of programs to prevent gender violence and gender-neutral values with in the context of education, especially at university levels.

\section{palabras clave/keywords:}

Violencia de género. Nivel académico. Género.

Gender violence. Scholar level. Gender.

\section{Introducción}

La problemática de la violencia de género está muy presente en todas las sociedades desarrolladas, tanto por su incidencia como por sus repercusiones a diferentes niveles - salud, legales, educacionales, sociales, económicas -, lo cual ha generado la puesta en marcha de programas preventivos, de reeducación de maltratadores, de atención a la víctima, entre otros, y ha sido objeto de estudio en multitud de trabajos empíricos. Son tan graves sus repercusiones sobre la mujer que se ha convertido en la principal causa de pérdida de años de vida en las mujeres entre 15 y 44 años (Amnistía Internacional, 2002). Su presencia se da en todos los ámbitos y contextos sociales, familiares, laborales y educativos (Menéndez, Pérez y Lorente, 2013; Sanmartín, Iborra, García y Martínez, 2010). 
Las investigaciones sobre la misma en parejas jóvenes, constatan que ésta se da a edades muy tempranas, en el caso de las mujeres a los 13 años y de los hombres alrededor de los 16 años (Rodríguez-Franco et al, 2012), produciéndose en muchos casos en la etapa de noviazgo en parejas adolescentes (Samaniego y Freixas, 2010). Otro dato a considerar es su incidencia, según Muñoz-Rivas, Graña, O’Leary y González (2006) la violencia física en relaciones en las que al menos uno de ellos es universitario, se sitúa alrededor del $20 \%$, y más de la mitad ha sufrido violencia psicológica. Valls (2008) habla de un 13\%, llegando al $65 \%$ cuando se analizan las conductas consideradas como formas de maltrato. Paino et al. (2011) la sitúa entorno al 6\% en adolescentes, mientras que un 12\% habría sufrido maltrato encubierto no reconocido y Vázquez, Torres, Otero, Blanco y López (2010) encuentran que un $6.3 \%$ de las universitarias españolas habría sufrido maltrato psicológico por su pareja. Blázquez, Moreno y García-Bahamonde (2009) lo sitúa en un 37\% con respecto a la indiferencia como forma de maltrato psicológico, siendo los universitarios entre 17 y 18 años, junto con los de 21 y 22 años los que presentan mayores conductas de maltrato psicológico como descalificaciones, trivializaciones, conductas destructivas y aislamiento social.

Según Meras (2003) un 40\% de las adolescentes relatan situaciones de violencia en su entorno frente al 34\% de los varones. Un estudio del Gobierno de Cantabria (2007) destaca que el $23 \%$ de las adolescentes con pareja presentaban entre 3 y 8 indicadores de violencia física o psicológica en su relación actual. Los estudios también apuntan otro dato preocupante, su normalización dentro de la relación de pareja, y su reciprocidad en parejas jóvenes (Rodríguez-Franco, et al., 2012; González-Ortega, Echeburúa y Corral, 2008).

El no reconocimiento de las situaciones de maltrato psicológico frente al maltrato físico (Samaniego y Freixas, 2010; Valls, 2008; Meras, 2003) dificulta conocer los casos reales de violencia, en especial cuando hablamos de parejas jóvenes, unido a la respuesta de la víctima de no denunciar, según Valls (2008) el 66\% de las universitarias que habían padecido este tipo de situaciones no denunciaron y actuaron contándoselo a alguien, o manteniéndolo en secreto.

Además, hemos de tener en cuenta la justificación que se hace del empleo de la violencia. Díaz Aguado, Martínez Arias y Martín Seoane (2004) y Viniegra (2007) los varones justifican su uso en mayor medida que las mujeres, en cambio las preadolescentes presentan mayor rechazo hacia actitudes y conductas sexistas (Ferragut, Blanca y Ortíz-Tallo, 2013; Lemus, Castillo, Moya, Padilla y Ryan, 2008).

Conocer su incidencia, la percepción con respecto a qué se considera maltrato y que no y el reconocimiento de los diferentes tipos, la justificación del uso de la violencia en la relación en adolescentes y jóvenes, puede servirnos para predecir cómo las nuevas generaciones responderán a esta situación, siendo un punto de reflexión acerca de cómo estamos educando y ayudarnos en el diseño de programas de prevención de la violencia contra las mujeres desde diferentes ámbitos educativos.

El presente trabajo ofrece una comparación entre la incidencia de la violencia de género, las conductas que la definen, la forma de actuar ante la misma y su justificación entre estudiantes de distintos niveles educativos, y el género. 


\section{Método}

\section{Participantes}

La muestra está constituida por 247 estudiantes, de los que 133 están cursando estudios universitarios en la Universidad de Valencia y 114 pertenecientes a otros niveles educativos, de estos, 45 estudian ESO (Enseñanza Secundaria Obligatoria), 51 Bachillerato y 18 PCPI (Programas de Cualificación Profesional) matriculados en dos institutos de secundaria de la ciudad de Valencia. Con respecto al género 165 son mujeres y 82 hombres. Las edades comprenden entre los 13 y los 25 años.

\section{Instrumento y variables}

Se ha empleado la "Encuesta de Violencia de Género en el Ámbito Educativo", elaborada para el presente estudio consta de un total de 32 ítems, algunos de los cuales son de respuesta única y otros de respuesta múltiple, la cual ha sido revisada por cinco expertos en violencia de género. Con vistas a determinar el índice de concordancia entre jueces se calculó el índice de Kappa, alcanzando un consenso entre jueces de .85, siendo este bastante bueno atendiendo a los índices de concordancia de Altman (1991).

Las variables seleccionadas para el presente trabajo fueron: Incidencia de violencia de género en el contexto educativo y en el contexto próximo, en las que el entrevistado ha de responder a la pregunta de si conoce algún caso de violencia de género bien en el contexto educativo, bien en su entorno más próximo - familiar, amistades o de pareja -.

Formas de actuar en caso de sufrir una situación de violencia de género dentro del contexto educativo. En esta variable el sujeto responde a cuatro situaciones que pudieran darse por parte de su pareja o expareja, dando una respuesta única entre las siguientes categorías denunciarlo, comunicarlo a un profesor, padres o amigos, mantenerlo en secreto o dejar la relación -.Estas situaciones son: 1. Sufrir empujones, zarandeos, besos y caricias sin consentimiento. 2. Desvalorizaciones, menosprecios, chantajes emocionales o coacciones para mantener relaciones afectivas o sexuales. 3. Que te prohíban tener relaciones de amistad o que invadan tu intimidad leyendo tus mensajes, correos... 4. Sentirte vigilado-a o perseguido-a, que te exijan obediencia, amenazas o intimidaciones.

La variable Percepción de la importancia de la violencia de género en la sociedad, presenta cuatro categorías de respuesta -nada importante, poco importante, importante y muy importante -.

A la hora de valorar la percepción sobre el maltrato, se incluyen los siguientes factores: Percepción del maltrato físico, definido por los ítems - Golpear a la pareja o expareja. Empujar y pellizcar -.Percepción del maltrato psicológico, el cual incluye - Humillaciones y desvalorizaciones como mujer. Hacer observaciones desagradables sobre la apariencia física. Sentir incomodidad o miedo por comentarios, llamadas, insistiendo mantener relaciones. Castigar o maltratar a los hijos cuando está enfadado contigo. Contar rumores respecto a la vida sexual o aspectos íntimos. Invadir la intimidad de la persona leyendo sus mensajes,

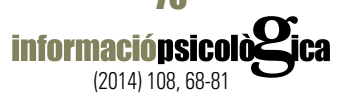


diario. Exigir obediencia. Perseguir insistentemente a la pareja o expareja. Amenazar, intimidar. Comparar con otras mujeres para desvalorizarte. Romper cosas de la pareja. Chantajear con contar secretos de la pareja o expareja. Amenazar con matarse o matar a los hijos si la pareja lo abandona. Hacer sentir inferior a la pareja o expareja. Culpabilizar a la pareja de sus desgracias -.Percepción del maltrato social, definido por - Vigilar o perseguir a la pareja o expareja. Prohibir o coaccionar a la hora de tener relaciones de amistad. Que la pareja exija saber con quién y dónde vas. Prohibir o coaccionar para dejar de estudiar, hacer deporte. Controlar la forma de vestir, peinarse de la pareja -. Percepción del maltrato económico - Controlar los ingresos económicos del hogar o de la familia -. Percepción del maltrato sexual, definido por los ítems - Presiones o uso de la fuerza para mantener relaciones sexuales. Intentar mantener relaciones sexuales contra su voluntad -. Las respuestas a estos ítems son dicotómicas, "sí lo considero violencia de género" al que se le asigna un valor de 1 o "no lo considero violencia de género" que se le asigna un valor de 0 . Se obtiene una puntuación global de cada uno de los tipos de maltrato sumando los ítems que la persona marca como afirmativos. La variable Percepción del maltrato global está formada por el conjunto de los 25 ítems que definen los tipos de maltrato que hemos enumerado anteriormente, obteniéndose una puntuación total al sumar sus puntuaciones.

Para la variable Aprobación del uso de la violencia física dentro de la relación de pareja, la respuesta es dicotómica, sí o no lo aprobaría. Los entrevistados han de responder a la pregunta si pueden pensar en alguna situación en la que aprobarían que su pareja les diese una bofetada.

La variable Conductas toleradas por amor, los encuestados han de responder sí tolerarían o no por amor a su pareja, en una sola ocasión, alguna de las siguientes conductas: Que me sea infiel. No me deje salir con amigos-as. Me prohíba hablar con otras personas. Que sea celoso-a. Controle mi forma de vestir o de comportarme. Me empuje. Me insulte. Me dé un bofetón. Me siga para ver que hago. Las respuestas a estos ítems son dicotómicas, "sí lo toleraría" al que se le asigna un valor de 1 o "no lo toleraría" que se le asigna un valor de 0 . La variable Tolerancia por amor queda definida por la suma de las puntuaciones en los ítems anteriores.

\section{Procedimiento}

Primeramente se pasó a la elaboración de la encuesta y su posterior valoración por diferentes expertos en la materia. En aquellos casos en los que los encuestados eran menores de edad se obtuvo la autorización de los padres o tutores antes de ser administrada. La encuesta se rellenaba de forma voluntaria y anónima, siendo el tiempo empleado para la misma de unos 10 minutos. En el caso de los universitarios la encuesta era rellenada online, en este caso para garantizar el anonimato del encuestado, una vez rellenada, se generaba un número único interno por encuesta y persona, borrando el programa los datos de usuario del encuestado. Todo esto se gestionó a través del equipo de informáticos de la Facultad de Psicología y de la Universidad de Valencia. Para el caso de los adolescentes, ésta se rellenó en formato

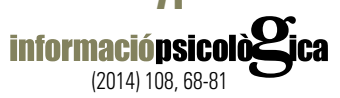


papel dentro del aula, con el apoyo de una de las psicólogas del equipo de investigación, en estos casos la encuesta era recogida indicando solamente edad, sexo y curso, sin recoger ningún dato referente a su nombre o iniciales.

Los institutos que participaron en el estudio fueron seleccionados porque previamente habían aceptado la puesta en marcha de un programa de prevención de la violencia de género en adolescentes en su centro. En cada uno de ellos se seleccionó un grupo de estudiantes perteneciente a cada nivel educativo, ESO, Bachillerato y PCPI, según la predisposición de los-as tutores-as.

Previo al pase de la encuesta, se informó a los padres sobre el objeto de estudio y el tratamiento confidencial de los datos a través de una carta, obteniéndose el correspondiente consentimiento informado.

Todos estos datos se recogieron entre abril y junio del 2013. Una vez obtenidos los datos se pasó al análisis estadístico y a su interpretación posterior.

\section{Análisis estadístico}

Para la comparación de ambos grupos se han realizado tablas de contingencia y pruebas $\chi 2$ en las variables categóricas, en los casos de baja frecuencia en alguna de las categorías hemos empleado la corrección de Monte Carlo y el estadístico exacto de Fisher. Se realizaron análisis de varianza multivariante (MANOVA) utilizando como factores fijos el nivel educativo y el género y como variables dependientes los factores de percepción del tipo de maltrato. Se empleó la Traza de Pillai para evaluar la significación de los efectos, siendo este estadístico más robusto al alejamiento de los supuestos del MANOVA (Tabachnick y Fidell, 2007). En el caso de darse efectos estadísticamente significativos se realizaron ANOVAS de continuación. El tamaño del efecto se comprobó mediante la eta-cuadrado parcial, siguiendo los estándares de Cohen (1992), interpretando estos efectos como bajo (sobre .02 ), medio (sobre .13) o alto (sobre .26). Se realizaron análisis de varianzas univariados (ANOVAS) en la comparación de medias para el género y el nivel educativo en función de las variables maltrato y tolerancia. Previo a estos análisis se comprobó que se cumplieran los supuestos de normalidad y homogeneidad de varianzas. En todos los análisis el intervalo de confianza ha sido del 95\%. Se ha utilizado el programa estadístico SPSS 19.

\section{Resultados}

\section{Incidencia de la violencia de género en los contextos de desarrollo}

Por lo que respecta al contexto educativo, un $13.5 \%$ de los universitarios dice conocer algún caso de violencia de género, frente al $22.8 \%$ de estudiantes de otros niveles educativos. Estas diferencias no resultaron ser estadísticamente significativas con un valor de $\chi 2$ (1) $=3.605, \mathrm{p}=.058, \theta=.121$.En relación al género no aparecen diferencias significativas con un valor de $\chi^{2}(1)=.046, p=.830, \theta=-.014$. Un $18.2 \%$ de las mujeres y un $17.1 \%$ de los hombres dice conocer algún caso de violencia de género dentro del ámbito educativo. 
En cuanto a la incidencia en el entorno próximo de la persona, no existen diferencias estadísticamente significativas entre ambos grupos de estudiantes, con un valor de $\chi 2(1)=$ $.166, \mathrm{p}=.683, \theta=-.026$. Destacar el porcentaje de casos donde este tipo de violencia está presente, en concreto en un $37.6 \%$ de universitarios y en un $35.1 \%$ en el caso de los no universitarios. Por lo que respecta al género no aparecen diferencias significativas con un valor de $\chi 2(1)=2.724, p=.099, \theta=-.105$. El 40\% de las mujeres reconocen una situación de violencia de género en su entorno próximo frente al $29.3 \%$ de los hombres.

\section{Formas de actuar ante situaciones de violencia de género}

En el caso de sufrir por parte de su pareja o expareja empujones, zarandeos, besos y caricias sin consentimiento, existen diferencias estadísticamente significativas según el nivel educativo con un valor de $\chi 2(3)=26.223, p=.000 . \theta=.329$. La respuesta que en mayor medida dan los universitarios es dejar la relación, mientras que en el caso de los no universitarios denunciar y comunicarlo.

Según el género no aparecen diferencias significativas $\chi 2(3)=2.150, p=.542 . \theta=.094$. Mientras que en los hombres predominan las respuestas de dejar la relación, las mujeres optan en mayor medida por dejar la relación o denunciar.

En los casos de sufrir desvalorizaciones, menosprecios, chantajes emocionales o coacciones para mantener relaciones afectivas o sexuales aparecen diferencias estadísticamente significativas tanto para la variable nivel educativo como el género, siendo los valores de $\chi 2(3)=31.447, \mathrm{p}=.000 . \theta=.358$ y $\chi 2(3)=10.913, \mathrm{p}=.012 . \theta=.211$ respectivamente. Mientras que los universitarios escogen como principales medidas a tomar denunciar y dejar la relación, los no universitarios eligen denunciar y comunicarlo. En función del género los hombres dejarían la relación como primera opción y las mujeres denunciarían.

Si la conducta por parte de la pareja o expareja fuese la de prohibir tener relaciones de amistad con otros compañeros o invadir su intimidad leyendo sus mensajes, correos, agenda, los no universitarios elegirían en mayor medida comunicarlo mientras que los universitarios dejar la relación. Estas diferencias resultaron ser estadísticamente significativas con un valor de $\chi 2(3)=29.397, p=.000 . \theta=.346$. Con respecto al género, tanto hombres como mujeres dejarían la relación, no habiendo diferencias significativas entre ambos gru$\operatorname{pos} \chi 2(3)=3.886, \mathrm{p}=.274 . \theta=.126$.

Ante la situación de sentirse vigilado-a o perseguido-a, que te exijan obediencia, amenazas o intimidaciones, los universitarios denunciarían en un mayor número de casos, seguido por dejar la relación y comunicarlo en porcentajes similares, los no universitarios en cambio eligen denunciar principalmente y comunicarlo, dándose diferencias significativas entre ambos grupos $\chi 2(3)=14.800, p=.002 . \theta=.246$. Según el género aparecen diferencias significativas $\chi 2(3)=15.628, p=.001 . \theta=.253$, tanto hombres como mujeres eligen en mayor medida la respuesta de denunciar frente a otras alternativas. Las frecuencias y porcentajes para cada una de las situaciones planteadas se pueden consultar en la tabla 1.

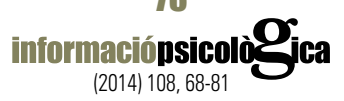




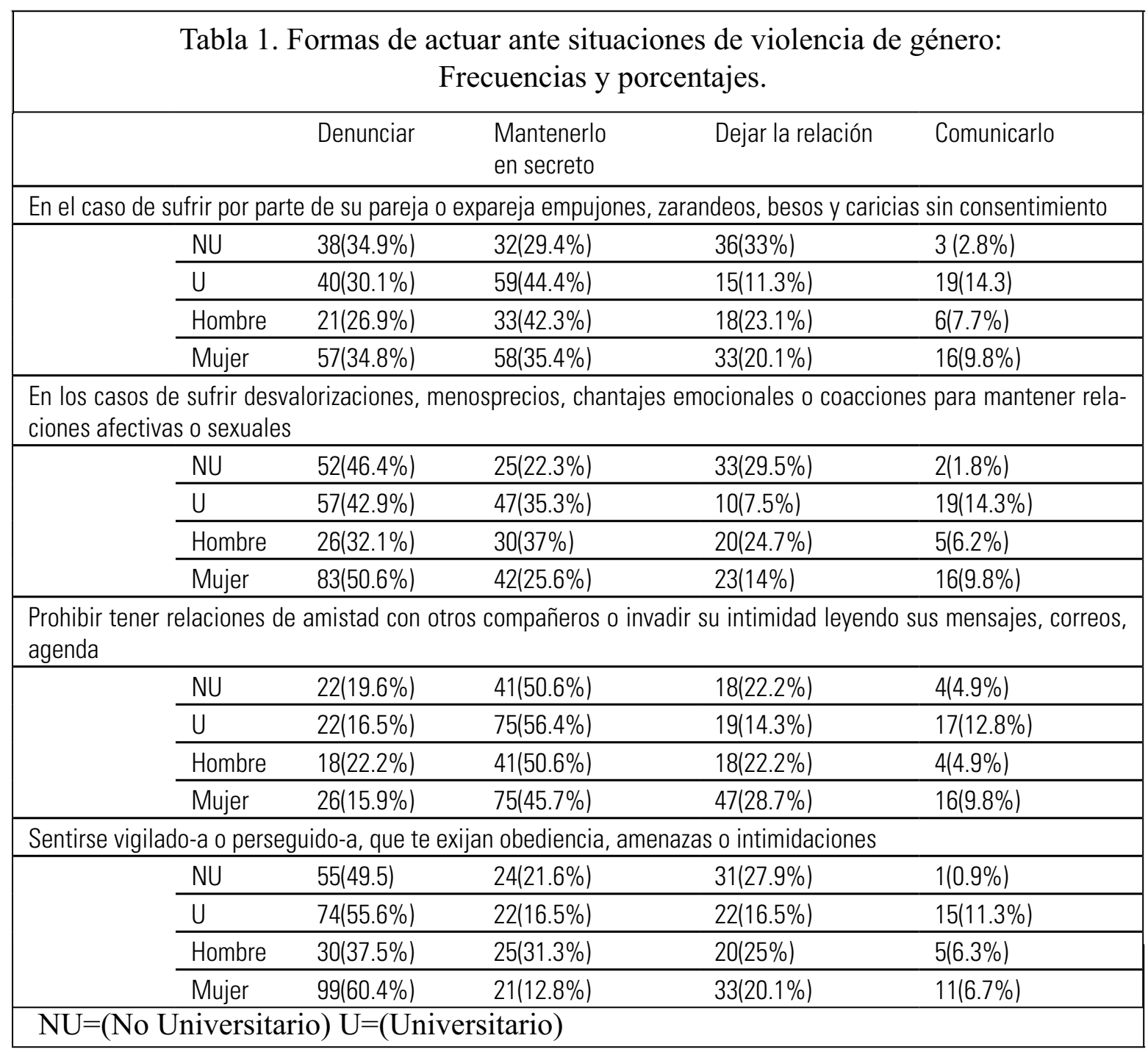

\section{Importancia de la violencia de género en la sociedad}

Con respecto al grado de importancia que la violencia de género tiene en la sociedad, son los universitarios los que en mayor medida consideran ésta como muy importante, en concreto un $75.9 \%$ frente a un $57 \%$ de no universitarios. En la categoría de importante los porcentajes para universitarios son del $21.8 \%$ frente al $40.4 \%$ de los no universitarios. Para el resto de categorías los porcentajes no llegan al 3\% en ambas muestras. Estas diferencias resultaron ser estadísticamente significativas, siendo el valor de $\chi 2(3)=13.278, p=.004$. $\theta$ $=.232 \mathrm{y}$ el estadístico exacto de Fisher $=12.787$ y la significatividad según la prueba de Montecarlo $\mathrm{p}=.002$.

Las diferencias según el género resultaron ser estadísticamente significativas siendo el valor de $\chi 2(3)=7.431, p=.059 . \theta=.173$ y el estadístico exacto de Fisher $=7.539$ y la significatividad según la prueba de Montecarlo $\mathrm{p}=.034$.El $72.1 \%$ de las mujeres lo considera muy importante frente al $57.3 \%$ de los hombres, importante en un $26.7 \%$ en las mujeres y un $37.8 \%$ 
en los hombres, en las categorías de nada y poco importante los porcentajes no superan el $1 \%$ en el caso de las mujeres y en el de los hombres el $3.7 \%$.

\section{Percepción sobre los diferentes tipos de maltrato}

Los análisis multivariados muestran que existe relación entre el tipo de maltrato y el género siendo estas diferencias estadísticamente significativas $\left(\mathrm{F}(5,239)=2.891 ; \mathrm{p}=.015, \mathrm{~h}^{2}=\right.$ $.057)$ y con respecto al nivel educativo $\left(\mathrm{F}(5,239)=14.743 ; \mathrm{p}=.000, \mathrm{~h}^{2}=.236\right)$, mientras que no habría relación en la interacción género y nivel educativo $(\mathrm{F}(5,239)=1.174 ; \mathrm{p}=$ $\left..322, \mathrm{~h}^{2}=.024\right)$.

Los ANOVA de continuidad respecto a la variable género permite conocer en qué factores del maltrato existen diferencias significativas, en maltrato psicológico $(\mathrm{F}(1,243)=5.103 ; \mathrm{p}$ $\left.=.025, \mathrm{~h}^{2}=.021\right)$ y en maltrato social $\left(\mathrm{F}(1,243)=7.654 ; \mathrm{p}=.006, \mathrm{~h}^{2}=.031\right)$, siendo las mujeres las que presentan una puntuación media mayor en el reconocimiento de estos tipos de maltrato (ver tabla 2). No existen diferencias en cuanto al maltrato físico $(\mathrm{F}(1,243)=1.503$; $\left.\mathrm{p}=.221, \mathrm{~h}^{2}=.006\right)$, al maltrato sexual $\left(\mathrm{F}(1,243)=.034 ; \mathrm{p}=.854, \mathrm{~h}^{2}=.000\right)$ y con respecto al maltrato económico $\left(\mathrm{F}(1,243)=2.013 ; \mathrm{p}=.157, \mathrm{~h}^{2}=.008\right)$.

Los efectos inter-sujetos muestran diferencias estadísticamente significativas en nivel educativo en los factores maltrato físico $\left(\mathrm{F}(1,243)=16.923 ; \mathrm{p}=.000, \mathrm{~h}^{2}=.065\right)$, maltrato psicológico $\left(\mathrm{F}(1,243)=25.363 ; \mathrm{p}=.000, \mathrm{~h}^{2}=.095\right)$, maltrato sexual $(\mathrm{F}(1,243)=15.661 ; \mathrm{p}$ $\left.=.000, \mathrm{~h}^{2}=.061\right)$, maltrato social $\left(\mathrm{F}(1,243)=46.914 ; \mathrm{p}=.000, \mathrm{~h}^{2}=.162\right)$ y maltrato económico $\left(\mathrm{F}(1,243)=44.230 ; \mathrm{p}=.000, \mathrm{~h}^{2}=.154\right)$, siendo las puntuaciones medias mayores para los estudiantes universitarios frente a los no universitarios tal y como se puede comprobar en la tabla 2.

\begin{tabular}{|lllllll|}
\hline \multicolumn{7}{|c|}{ Tabla 2. Descriptivos según los diferentes tipos de maltrato en función del nivel } \\
educativo y del género. \\
\hline Variable & Nivel educativo & Media & DT & Género & Media & DT \\
\hline Maltrato físico & $\mathrm{U}$ & 1.84 & 0.35 & Hombre & 1.75 & 0.43 \\
& $\mathrm{NU}$ & 1.59 & 0.51 & Mujer & 1.72 & 0.46 \\
\hline Maltrato psicológico & $\mathrm{U}$ & 12.33 & 3.09 & Hombre & 10.20 & 4.20 \\
& $\mathrm{NU}$ & 9.78 & 3.88 & Mujer & 11.63 & 3.33 \\
\hline Maltrato sexual & $\mathrm{U}$ & 1.96 & 0.19 & Hombre & 1.86 & 0.37 \\
& $\mathrm{NU}$ & 1.78 & 0.49 & Mujer & 1.88 & 0.37 \\
\hline Maltrato social & $\mathrm{U}$ & 4.19 & 1.25 & Hombre & 3.00 & 1.81 \\
& $\mathrm{NU}$ & 2.73 & 1.81 & Mujer & 3.78 & 1.57 \\
\hline Maltrato económico & $\mathrm{U}$ & 0.66 & 0.47 & Hombre & 0.36 & 0.48 \\
& $\mathrm{NU}$ & 0.22 & 0.42 & Mujer & 0.51 & 0.50 \\
\hline Maltrato global & $\mathrm{U}$ & 21.01 & 4.57 & Hombre & 17.19 & 6.41 \\
& $\mathrm{NU}$ & 16.12 & 6.04 & Mujer & 19.53 & 5.36 \\
\hline NU=(No Universitario) $\mathrm{U}=($ Universitario) & & & & \\
& & & & & & \\
\hline
\end{tabular}




\section{Aprobación y tolerancia de conductas relacionadas con la violencia de género.}

A la pregunta si existiría alguna situación en que aprobarían que su pareja les diese una bofetada, aparecen diferencias estadísticamente significativas entre universitarios y estudiantes de otros niveles educativos, con una $\chi 2(1)=26.697, p=.000 . \theta=.338$. Son los estudiantes de otros niveles los que en mayor medida sí encuentran una situación en la que aprobarían que su pareja les diese una bofetada, en concreto en un $43.3 \%$ frente a un $56.7 \%$ que no, y en el caso de los universitarios un $13.2 \%$ frente al $86,8 \%$ que considera que no. En relación con el género aparecen diferencias estadísticamente significativas con una $\chi 2(1)$ $=19.855, \mathrm{p}=.000 . \theta=.292$, siendo los hombres los que en mayor medida justifican el uso de la violencia en la relación frente a las mujeres, en concreto un $45.3 \%$ de hombres sí que lo aprobarían frente a un $54.7 \%$ que no, y entre las mujeres un $17.7 \%$ sí lo aprobaría y un $82.3 \%$ no.

Para la variable Tolerancia el ANOVA muestra diferencias estadísticamente significativas para la variable género $\left(\mathrm{F}(1,241)=5.094 ; \mathrm{p}=.025, \mathrm{~h}^{2}=.021\right)$, siendo los hombres los que presentan una puntuación media mayor $(\mathrm{M}=2.96$, $\mathrm{DT}=2.45)$ frente a las mujeres $(\mathrm{M}=$ $1.98, \mathrm{DT}=2.41)$ y con respecto al nivel educativo $\left(\mathrm{F}(1,241)=36.349 ; \mathrm{p}=.000, \mathrm{~h}^{2}=.131\right)$, siendo los no universitarios los que presentan una mayor tolerancia $(\mathrm{M}=3.32$, $\mathrm{DT}=2.54)$ frente a los universitarios $(\mathrm{M}=1.46$, $\mathrm{DT}=2.05)$, no habiendo diferencias en la interacción del género con el nivel educativo $\left(\mathrm{F}(1,241)=1.196 ; \mathrm{p}=.275, \mathrm{~h}^{2}=.005\right)$.

Si nos fijamos en la variable Conductas que tolerarían por amor, de nuevo encontramos diferencias significativas según el nivel educativo en todos los indicadores menos el que hace referencia a la infidelidad, y siempre son los estudiantes de niveles educativos distintos al universitario los que en mayor medida tolerarían estas situaciones. Con respecto al género aparecen diferencias estadísticamente significativas en los indicadores - que controle mi forma de vestir, que me empuje y que me dé un bofetón -, siendo los varones los que en mayor medida tolerarían estas situaciones. Todos estos datos se pueden consultar en las tablas 3 y 4.

Tabla 3. Resultados en la comparación según el nivel educativo y el género en relación a la variable tolerancia por amor.

\begin{tabular}{|lllllllll|}
\hline \multicolumn{7}{l}{ Nivel educativo } & \multicolumn{7}{l|}{ Género } \\
\hline & $\chi 2$ & g.l. & $\mathrm{p}$ & $\theta$ & $\chi 2$ & $\mathrm{~g}$. l. & $\mathrm{p}$ & $\theta$ \\
\hline Me sea infiel & 0.205 & 1 & .651 & .029 & 0.256 & 1 & .613 & -.032 \\
\hline No me deje salir con amigos-as & 28.521 & 1 & $.000^{*}$ & .341 & 0.815 & 1 & .367 & .058 \\
\hline $\begin{array}{l}\text { Me prohíba hablar con los-as } \\
\text { amigos-as }\end{array}$ & 21.828 & 1 & $.000^{*}$ & .298 & 1.306 & 1 & .235 & .073 \\
\hline Que sea celoso-a & 29.174 & 1 & $.000^{*}$ & .345 & 1.075 & 1 & .300 & .066 \\
\hline Controle mi forma de vestir & 14.178 & 1 & $.000^{*}$ & .241 & 9.593 & 1 & $.002^{*}$ & .198 \\
\hline Me empuje & 20.631 & 1 & $.000^{*}$ & .290 & 29.545 & 1 & $.000^{*}$ & .347 \\
\hline Me insulte & 6.939 & 1 & $.008^{*}$ & .168 & 3.547 & 1 & .060 & .120 \\
\hline Me dé un bofetón & 17.450 & 1 & $.000^{*}$ & .267 & 18.721 & 1 & $.000^{*}$ & .276 \\
\hline Me siga para ver que hago & 9.474 & 1 & $.002^{*}$ & .197 & 1.485 & 1 & .223 & .078 \\
\hline No toleraría estas situaciones & 25.436 & 1 & $.000^{*}$ & -.322 & 3.285 & 1 & .070 & -.116 \\
\hline$*$ p $<.05$ & & & & & & & & \\
\hline
\end{tabular}




\begin{tabular}{|c|c|c|c|c|c|c|}
\hline \multicolumn{7}{|c|}{$\begin{array}{l}\text { Tabla 4. Porcentajes de tolerancia por amor en función } \\
\text { del nivel educativo y el género. }\end{array}$} \\
\hline Variables & Grupo & Sí & No & Grupo & $\overline{\text { Sí }}$ & No \\
\hline \multirow[t]{2}{*}{ Me sea infiel } & $\mathrm{U}$ & $33.8 \%$ & $66.2 \%$ & Hombre & $32.9 \%$ & $67.1 \%$ \\
\hline & $\mathrm{NU}$ & $36.6 \%$ & $63.4 \%$ & Mujer & $36.2 \%$ & $63.8 \%$ \\
\hline \multirow[t]{2}{*}{ No me deje salir con amigos-as } & $\mathrm{U}$ & $12.0 \%$ & $88.0 \%$ & Hombre & $29.3 \%$ & $70.7 \%$ \\
\hline & $\mathrm{NU}$ & $42.0 \%$ & $58.0 \%$ & Mujer & $23.9 \%$ & $76.1 \%$ \\
\hline \multirow[t]{2}{*}{ Me prohíba hablar con amigos-as } & $\mathrm{U}$ & $12.0 \%$ & $88.0 \%$ & Hombre & $28.0 \%$ & $72.0 \%$ \\
\hline & $\mathrm{NU}$ & $37.5 \%$ & $62.5 \%$ & Mujer & $21.5 \%$ & $78.5 \%$ \\
\hline \multirow[t]{2}{*}{ Que sea celoso-a } & $\mathrm{U}$ & $36.8 \%$ & $63.2 \%$ & Hombre & $57.3 \%$ & $42.7 \%$ \\
\hline & $\mathrm{NU}$ & $71.4 \%$ & $28.6 \%$ & Mujer & $50.3 \%$ & $49.7 \%$ \\
\hline \multirow[t]{2}{*}{ Controle mi forma de vestir } & $U$ & $7.5 \%$ & $92.5 \%$ & Hombre & $25.6 \%$ & $74.4 \%$ \\
\hline & $\mathrm{NU}$ & $25.0 \%$ & $75.0 \%$ & Mujer & $10.4 \%$ & $89.6 \%$ \\
\hline \multirow[t]{2}{*}{ Me empuje } & $\mathrm{U}$ & $9.0 \%$ & $91.0 \%$ & Hombre & $39.0 \%$ & $61.0 \%$ \\
\hline & $\mathrm{NU}$ & $32.1 \%$ & $67.9 \%$ & Mujer & $9.8 \%$ & $90.2 \%$ \\
\hline \multirow[t]{2}{*}{ Me insulte } & $\mathrm{U}$ & $7.5 \%$ & $92.5 \%$ & Hombre & $18.3 \%$ & $81.7 \%$ \\
\hline & $\mathrm{NU}$ & $18.8 \%$ & $81.3 \%$ & Mujer & $9.8 \%$ & $90.2 \%$ \\
\hline \multirow[t]{2}{*}{ Me de un bofetón } & $\mathrm{U}$ & $4.5 \%$ & $95.5 \%$ & Hombre & $25.6 \%$ & $74.4 \%$ \\
\hline & $\mathrm{NU}$ & $22.3 \%$ & $77.7 \%$ & Mujer & $6.1 \%$ & $93.9 \%$ \\
\hline \multirow[t]{2}{*}{ Me siga para ver que hago } & $\mathrm{U}$ & $12.8 \%$ & $87.2 \%$ & Hombre & $24.4 \%$ & $75.6 \%$ \\
\hline & $\mathrm{NU}$ & $28.6 \%$ & $71.4 \%$ & Mujer & $17.8 \%$ & $82.2 \%$ \\
\hline \multirow[t]{2}{*}{ No toleraría estas situaciones } & $\mathrm{U}$ & $42.9 \%$ & $57.1 \%$ & Hombre & $22.0 \%$ & $78.0 \%$ \\
\hline & $\mathrm{NU}$ & $13.4 \%$ & $86.6 \%$ & Mujer & $33.1 \%$ & $66.9 \%$ \\
\hline
\end{tabular}

\section{Discusión}

La violencia de género se manifiesta por igual en el ámbito educativo universitario como no universitario, así como en los contextos próximos de los estudiantes, como son el familiar, su propia relación de pareja o en las relaciones de pareja que mantienen sus amistades. Destacar la incidencia de la misma, tanto en el mismo contexto educativo, entre un $13 \%$ y un $22 \%$, y fuera del mismo, entre un $35 \%$ y un $37 \%$, por lo que la exposición a este tipo de violencia entre los estudiantes entre 13 y 25 años es alta, coincidiendo con los resultados obtenidos en otros estudios (Muñoz-Rivas et al., 2006; Valls, 2008; Gobierno de Cantabria, 2007). Con respecto al género, es entre las mujeres las que más situaciones de violencia de género se dan en su entorno, tal y como apunta Meras (2003), circunstancia que puede estar relacionada con la identificación de las conductas que definen los tipos de maltrato, al ser estas las que en mayor medida las identifican frente a los hombres, principalmente en lo referido al maltrato psicológico y social. A su vez, son también los hombres los que en mayor medida justifican el uso de la violencia dentro de la relación de pareja, aspecto que coincide con los trabajos de Díaz Aguado et al., (2004) y Viniegra, (2007), y a la vez son las que más las toleran. 
El reconocimiento de las conductas relacionadas con los diferentes tipos de malos tratos es menor entre los estudiantes no universitarios, lo cual se da en todos los tipos de maltrato analizados. Esto, unido a una mayor justificación del empleo de la violencia en la relación de pareja y a una mayor tolerancia por amor de conductas relacionadas con la violencia física o psicológica, nos lleva a considerar a este grupo como más vulnerable o de mayor riesgo de sufrir situaciones de violencia dentro de la relación de pareja frente a los estudiantes universitarios.

Las respuestas ante situaciones de violencia suelen resolverse sin presentar denuncia, sobre todo en el grupo de los universitarios, que optan como medida dejar la relación frente a los estudiantes de otros niveles educativos, los cuales actuarían presentando una denuncia en mayor medida en tres de las cuatro situaciones que se exponen, y en segundo lugar la respuesta que se plantean es la de comunicarlos a alguien próximo, lo cual no garantiza el fin de la relación o que se tramite la correspondiente denuncia. Los tipos de respuestas ante el maltrato por la pareja pueden venir condicionadas por la edad de la víctima, en la medida en que los estudiantes universitarios, que tienen mayor edad, deciden ellos mismos solucionar el problema dejando la relación, se ven capaces y maduros para hacerlo, mientras que los estudiantes de niveles educativos no universitarios y mayoritariamente de menor edad su respuesta al maltrato está relacionada con la necesidad de comunicarlo. Esta tendencia a no denunciar confirma las aportaciones de otros trabajos como el de Valls (2008), lo que permite encubrir gran parte de situaciones de violencia de género, ya que sólo se denuncian un $10 \%$ de los casos reales, dejando ver sólo la punta del iceberg.

El no denunciar, la justificación o tolerancia de la violencia o el bajo reconocimiento de diferentes conductas relacionadas con el maltrato, pueden ser explicadas por la creencia de que se puede querer y maltratar a la vez, tal y como así refleja Meras (2003), encontrando que entre el $80 \%$ y el $75 \%$ de los adolescentes sostienen que se puede amar y agredir en una relación de pareja, o debido a justificaciones de la conducta agresiva por parte de la víctima como la pérdida de control pasajera por parte de su pareja. Otra situación preocupante, que puede explicar esta situación, y que ya ha sido apuntada en diferentes trabajos (Corral, 2009; Fernández-Fuertes y Fuertes, 2010), es la normalización y bidireccionalidad de la violencia entre las parejas más jóvenes.

Para concluir, podemos decir que los programas de prevención de la violencia de género y de relaciones de pareja saludables deben ser iniciados en la adolescencia, al ser este un periodo crítico donde se producen las primeras relaciones de pareja. Según González-Ortega et al. (2008) es en ese momento cuando la mujer tiene una mayor capacidad de poder elegir si continuar o no con la relación iniciada previamente, siendo en estas relaciones iniciales donde se aprenden las pautas de interacción de sumisión o de agresividad que perdurarán en las relaciones adultas. Además, tal y como señalan Sarasua, Zubizarreta, Echeburúa y Corral (2007) las consecuencias psicológicas de sufrir un maltrato por parte de la pareja son mayores a edades más jóvenes, lo cual justifica la puesta en marcha de este tipo de actua- 
ciones preventivas.

Diferentes autores señalan el ámbito escolar como contexto principal donde llevar a la práctica dichos programas, tal y como sugieren Hernando (2007) y Mañas Viejo (2010). En especial entre los estudiantes de Educación Secundaria Obligatoria como así también subraya De la Fuente, Peralta, y Sánchez Roda, (2006), al ser este uno de los grupos de mayor riesgo, tal y como indican los resultados presentados. Por otro lado, al ser esta etapa de escolarización obligatoria, garantizaría llegar a un mayor número de sujetos, ya que a partir de los 16 años algunos de ellos ya no continuarán dentro del sistema educativo, limitando en este sentido el acceso a estos programas.

Los programas de prevención de la violencia de género y de relaciones de pareja saludables han de abordar aspectos como las relaciones positivas y saludables en la relación de pareja y la solución de conflictos mediante estrategias asertivas, el cuestionamiento del uso de la violencia en general y en particular en el caso de las relaciones de pareja, el reconocimiento de los derechos de las personas con independencia de cualquier condición, en especial por el hecho de ser hombre o mujer, así como el cuestionamiento de los roles de género tradicionales y sexistas, principalmente en los varones (Ferragut, Blanca y Ortíz-Tallo, 2013), la identificación de los prejuicios que la sociedad tiene contra las víctimas (Montilla, Pérez, Castillo-Mayen y Montes-Berges, 2012), el reconocimiento de indicadores y conductas relacionadas con la violencia de género, el desarrollo de la autoestima, los recursos de atención y ayuda en caso de sufrir agresiones de cualquier tipo por parte de la pareja. Desde una concepción sistémica, estos programas de prevención de la violencia de género en adolescentes han de involucrar a los padres y madres de los adolescentes, en la medida en que valoren la transmisión de roles sexistas dentro del hogar, en las relaciones de pareja y en la forma diferencial de educar a hijos e hijas, así como proporcionar estrategias asertivas de resolución de conflictos. Por último, tampoco podemos desvincular de estas actuaciones al profesorado, especialmente y por la importancia que tiene a quienes ejercen las funciones de tutoría, contemplando estas actuaciones preventivas no solo de forma transversal en el currículum, sino también, como actividad dentro de la acción tutorial.

Entre las limitaciones del estudio hemos de considerar el tamaño reducido de la muestra, el tipo de muestreo realizado a la hora de seleccionar los centros educativos y el no contar con jóvenes mayores de 16 años que ya han superado la edad de escolarización obligatoria y se encuentran fuera del sistema educativo. Todos estos aspectos condicionan la generalización de los resultados obtenidos. Por otro lado, sería conveniente para continuar con la investigación en esta línea, la inclusión de grupos de jóvenes insertos en el mundo laboral o desempleados, así como determinar la influencia que pueden tener otras variables sobre la percepción de la violencia de género, como el nivel sociocultural o la cultura de origen, entre otras. 
Altman, D. G. (1991). Practical stadistics for medical research. New York: Chapman and Hall.

Amnistía Internacional (2002). Violencia de género en el ámbito familiar y protección de los derechos humanos de las mujeres en España, Informe de noviembre, 2002, Amnistía Internacional.

Blázquez, M., Moreno, J.M. y García-Baamonde, M.E. (2009). Estudio del maltrato psicológico, en las relaciones de pareja, en jóvenes universitarios.Electronic Journal of Research in Educational Psychology, 7(2), 691-714.

Cohen, J. (1992). A power primer. Psychological Bulletin, 112, 155-159.

Corral, S. (2009). Estudio de la violencia en el noviazgo en jóvenes universitarios/as: cronicidad, severidad y mutualidad de las conductas violentas. Psicopatología Clínica Legal y Forense, 9, 29-48.

De la Fuente, J., Peralta, F.J. y Sánchez Roda, M.D. (2006). Valores sociopersonales y problemas de convivencia en la Educación Secundaria. Electronic Journal of Research in Educational Psychology, 4(2), 171-200.

Díaz Aguado, M.J., Martínez Arias, R., y Martín Seaone, G. (2004). Prevención de la violencia y lucha contra la exc/usión desde la adolescencia. Vol. I. La violencia entre iguales en la escuela y en el ocio. Estudios comparativos e instrumentos de evaluación. Madrid: Instituto de la Juventud.

Fernández-Fuertes, A. y Fuertes, A. (2010). Physical and psychological aggression in dating relationships of Spanish adolescents: Motives and consequences. Child Abuse \& Neglect, 34(3), 183-191.

Ferragut, M., Blanca, Ma.J. y Ortíz-Tallo, M. (2013). Psychological values as protective factors against sexist attitudes in preadolescents. Psicothema, 15(1), 38-42. http://www.dx.doi.org/10.7334/psicothema2012.85

Gobierno de Cantabria (2007). Estudio sobre la violencia de género y actitudes sexistas en la población joven de Cantabria. Santander: Dirección General de la Mujer.

González-Ortega, I., Echeburúa, E. y Corral, P. (2008). Variables significativas en las relaciones violentas en parejas jóvenes: una revisión. Behavioral Psychology, 16(2), 207-225.

Hernando, A. (2007). La prevención de la violencia de género en adolescentes. Una experiencia en el ámbito educativo. Apuntes de Psicología, 25(3), 325-340.

Lemus, S., Castillo, E., Moya, M., Padilla, M. y Ryan, E. (2008). Elaboración y validación del Inventario de Sexismo Ambivalente para Adoles. centes. International Journal of Clinical and Health Psychology, 8(2), 537-562.

Mañas Viejo, C. (2010). Aspectos generales en la intervención psicopedagógica ante la violencia de género. En E. López-Zafra, (Coord.), Violencia contra las mujeres: Descripción e intervención biopsicosocial (pp. 207-227). Newbury Park: Sage Publicaciones.

Menéndez, S., Pérez, J. y Lorence, B. (2013). La violencia de pareja contra la mujer en España: Cuantificación y caracterización del problema, las víctimas, los agresores y el contexto social y profesional. Psychosocial Intervention, 22(1) 41-53. http://www. dx.doi.org/10.5093/in2013a6

Meras, A. (2003). Prevención de la violencia de género en adolescentes. Estudios de Juventud, 62(3), 143-150.

Montilla, G., Pérez, L. G., Castillo-Mayén, M.R. y Montes-Berges, B. (2012). Buscando la educación consciente de la problemática de la violencia de género: Diseño de una página web. Revista Magina

Muñoz Rivas, M., Graña, J. L., 0 `Leary, K. D. y González, P. (2006). Agresión física y psicológica en las relaciones de noviazgo en universitarios españoles. Psicothema, 19(1), 102-107.

Paino, S. et al. (2011). Violencia entre novios. Recuperado de http://www.infocop.es/view article.asp?id =3466

Rodríguez Franco, L., López Cepero, J., Rodríguez Díaz, F. J., Bringas, C., Estrada, C., Antuña, M. A. \& Quevedo-Blasco, R. (2012). Labeling dating abuse: Undetected abuse among Spanish adolescents and young adults. International Journal of Clinical and Health Psychology, 12, 55-67.

Samaniego, E. y Freixas, A. (2010).Estudio sobre la identificación y vivencia de violencia en parejas adolescents. Apuntes de Psicología, 28(3), 349-366. 


\section{temas de estudio}

Francisco González Sala y Belén Mora Valero

Sanmartín, J., Iborra, I., García, Y. y Martínez, P. (2010). III Informe internacional violencia contra las mujeres en las relaciones de pareja. Estadísticas y legislación. Valencia: Centro Reina Sofía para el Estudio de la Violencia.

Sarasua, B., Zubizarreta, I., Echeburúa, E. y Corral, P. (2007). Perfil psicopatológico diferencial de las víctimas de violencia de pareja en función de la edad. Psicothema, 19, 459-466.

Tabachnick, B. G. y Fidell, L. S. (2007). Using multivariate statistics, (5a ed.). Boston: Allyn and Bacon.

Valls, R. (2008). Violencia de género en las universidades españolas, Año 2006 -2008. Madrid: Ministerio de Igualdad.

Vázquez, E., Torres, A., Otero, P., Blanco, V. y López, M. (2010). Prevalencia y factores de riesgo de la violencia contra la mujer en estudiantes universitarias españolas. Psicothema, 22(2), 196-201.

Viniegra, M. (2007). Actitudes y creencias en torno a la violencia en adolescentes de Secundaria. Pulso, 30, 75- 11. 